\title{
INTERTEXTUALITÉ BIBLIQUE ET LA TRADUCTION
}

Iryna VYGNANSKA*

\section{Biblical Intertextuality and Translation}

\begin{abstract}
The notion of intertextuality is used to describe links between texts. To examine biblical intertextuality, we will adopt a narrower meaning of the term as a conventional reference to relations between different texts embodied in quotation, reference or allusion in order to consider a set of echoes, continuities and deviations. If we consider that every text is presented as a palimpsest that allows communication with the literary memory, any textuality would be interpreted as intertextuality. Intertextuality can take different forms, and allows the text to be considered as a living product containing a universal culture. A translator can intervene through explicit practices, sometimes risking to change the effect produced or targeted at. One possible solution is to stress the function of the intertextual links; another is adaptation. The article shows that the translated biblical text has its place, which is not the same as that of the original text. The translator must accept the actual reality of the translation, which may not be equal to the value of the original text. Intertextuality and translation have a close and very fruitful relationship.
\end{abstract}

Keywords: intertextuality, allusion biblical, reference, quatation, translation, Old and New Testament.

Dans les textes critiques l'intertextualité est définie par D. Maingueneau ${ }^{1}$ comme «l'ensemble de relations avec d'autres textes se manifestant à l'intérieur d'un texte». Le mot et la notion d'intertextualité apparaissent au sein du groupe d'avant-garde Tel Quel': «Tout texte se situe à la jonction de plusieurs textes dont il est à la fois la relecture, l'accentuation, la condensation, le déplacement et la profondeur». Kristeva et Barthes affirment qu' «un texte appelle à la mémoire du lecteur d'autres textes».

Julia Kristeva: «Tout texte se construit comme mosaïque de citations, tout texte est absorption et transformation d'un autre texte». Dans l'espace d'un texte plusieurs énoncés pris à d'autres textes se croisent et se

\footnotetext{
* Université Nationale Ivan Franko de Lviv, Faculté des Langues Etrangères, Philologie française, Universytetska St, 1, Lviv, L'vivs'ka oblast, UCRAINA, 79000

(irene_baytsar@yahoo.fr).

1 Maingueneau1976: 191

2 Foucault 1968:410
} 
neutralisent» ${ }^{3}$. Ainsi, l'auteur construirait son texte en exploitant des fragments de textes antérieurs. L'intertextualité n' est pas à proprement parler conçue comme un phénomène d'imitation: il s'agit moins de citer les textes antérieurs que d'en retrouver des traces, parfois disséminées inconsciemment par l'auteur. La notion est née de l'idée de «polyphonie», développée par Bakhtine, dont l'œuvre fut écrite dans les années 1920, mais ne fut traduite et interprétée en France que vers la fin des années 1960.

Julia Kristeva postule qu'aucun texte ne peut s'écrire indépendamment des écrits qui l'ont précédé. Dans ce contexte, l'intertextualité devient un vaste mouvement de répercussion infinie de textes en intertextes, à l'instar d'une réflexion illimitée générée par la disposition parallèle de miroirs ${ }^{4}$. La même vision est partagée par Barthes pour qui «Tout texte est un intertexte; d'autres textes sont présents en lui, à des niveaux variables, sous des formes plus ou moins reconnaissables (...) L'intertexte est un champ général de formules anonymes, dont l'origine est rarement repérable, de citations inconscientes ou automatiques" 5

$\mathrm{Au}$ cours de la décennie suivante, la notion est vite diffusée et fait l'objet d'un inventaire tous azimuts, où la matière transformée est un discours idéologique (Barthes), un contenu de l'inconscient (Kristeva), une phraséologie (Riffaterre). Pour Barthes, «tout texte est un intertexte; d'autres textes y sont présents sous des formes variables: les textes de la culture antérieure et ceux de la culture environnante; tout texte est un tissu de citations révolues. Distribuésdans le texte des morceaux de codes, des formules, des modèles rythmiques, des fragments de langages sociaux, etc., car il y a toujours du langage avant le texte et autour de lui».

La définition de G. Genette apporte une clarification qui est devenue l'objet d'un consensus. Selon Genette on distingue une intertextualité au sens étroit, définie par la «relation de coprésence entre deux ou plusieurs textes», «la présence effective d'un texte dans un autre» ${ }^{6}$. Prenant en considération la notion précédente, G. Genette étudie tous les faits d'intertextualité, qu'il a rebaptisée du nom plus large de transtextualité: elle est «tout ce qui met [le texte] en relation, manifeste ou secrète, avec d'autres textes». La transtextualité inclut quatre types de relations :

-L'architextualité: la relation qu'un texte entretient avec la catégorie générique à laquelle il appartient.

-La paratextualité: la relation d'un texte avec son paratexte (préfaces, avertissements...).

- La métatextualité: la relation de commentaire qui «unit un texte à

3 Kristeva 1969: 82-112

4 Kristeva 1969:146

5 Barthes 1993: 58-59

6 Genette 1982: 7-14 
un autre texte dont il parle, sans nécessairement le citer (le convoquer), voire, à la limite, sans le nommer. C' est par excellence la relation critique».

-L'intertextualité: elle comprend autant l'allusion, le plagiat que la citation. L'intertextualité peut ainsi prendre différentes formes, et permet de considérer le texte comme une production vivante qui contient un morceau de culture universelle.

La mise en relation peut concerner l'œuvre toute entière et son support peut se manifester sous forme d'unités discontinues, comme un motif narratif, une structure ou un style d'écriture. Son repérage se base sur l'étude dans lequel un emprunt est identifiable; on utilise les expressions «texte citant» pour le second texte et «texte cité» pour le texte préexistant, même si la «citation» n'est pas la seule forme d'emprunt possible.

Les définitions de l'intertextualité sont diverses; si la richesse du concept semble autoriser les interprétations les plus larges, un certain nombre d'études cédant à la mode de l'intertextualité, a un lien théorique pluslarge avec le concept.

Les critiques proposent la nouvelle notiont d'«intertextualité» dans une vieille critique de sources qui consiste, d'après Marc Angenot ${ }^{7}$, «à faire $\mathrm{du}$ neuf avec du vieux, à appeler analyse intertextuelle, une bien traditionnelle critique philologique des sources et des influences littéraires». Selon l'auteur l'idée d'intertextualité «sert à proclamer la bonne nouvelle de la mort du sujet».

«À la place de la notion d'intersubjectivité s'installe celle d'intertextualité» affirme Kristeva: ce sont les textes qui dialoguent, non les sujets, lesquels sont totalement expulsés des œuvres. Il nous semble que dans l'intertextualité, le sujet ne meurt pas mais s'affirme.

Pour Riffaterre «l'intertextualité est le mécanisme propre de la lecture littéraire. Tous les mots qui composent [le texte] n'auront de fonction littéraire que lorsqu'ils seron compris en fonction de l'intertexte qu'ils présupposent» ${ }^{8}$. M. Riffaterre explore depuis la fin des années $70 \mathrm{sa}$ théorie de l'intertextualité dans le cadre d'une théorie de la réception.

L'intertextualité ne demande pas de «prouver le contact entre l'auteur et ses prédécesseurs. Il suffit pour qu'il y ait intertexte que le lecteur fasse le rapprochement entre l'auteur et ses prédécesseurs». Il revient au lecteur de déterminer la «dose» d'intertextualité du texte: seul récepteur peut établir l'intertexte.

Le problème de l'intertextualité se présente parce que le récepteur établit l'intertexte qui peut varier d'un lecteur à l'autre, selon la culture et les lectures des lecteurs. Si la responsabilité du renvoi intertextuel incombe au lecteur, l'intertextualité est-elle acceptable? Il est impossible de définit

7 Angenot 1983: 121-135

8 Riffaterre 1979: 131 
un moment où l'intertexte est convoqué par l'auteur, inconsciemment provoqué par lui, ou redevable au lecteur.L'intertextualité est un phénomène de réception. C'est pourquoi il faut accepter le sens très large de l'intertextualité.

Une autre étude du concept de texte et d'intertexte, s'opère dans les domaines mythiques et historiques. En 1987, Marc Eigeldingerprécisait à ce sujet: «Mon projet est de ne pas limiter la notion d'intertextualité à la seule littérature, mais de l'étendre aux divers domaines de la culture. Elle peut être liée à l'émergence d'un autre langage à l'intérieur du langage littéraire; par exemple celui de la Bible ou de la mythologie, ainsi que celui de la philosophie» ${ }^{9}$. De fait, ce serait donc toute allusion à notre culture qui relèverait de l'intertextualité. On est loin de la primitive de l'intertexte, qui renvoyait à une œuvre antérieure - c'est le cas de la Bible, mais non de toute la tradition mythologique. La notion d'intertextualité, de «relation entre différents textes écrits», se dissout dans une définition plus large des références culturelles.

Dans la pratique concrète on définit deux tendances opposées. Certains, comme M. Riffaterre, s'attachent à étudier des allusions et des citations. La dissonance avec le reste du texte nécessite le recours à un intertexte. Ils focalisent leur attention sur la microstructure. D'autres, commeG. Genette,plutôt que d'étudier les microstructures ou les fragments, préféraient les macrostructures: les structures ou les traits génériques d'un texte.

Laurent Jenny distingue l'intertextualité proprement dite, comme «rapports de texte à texte en tant qu'ensembles structurés» ${ }^{10}$, et l'intertextualité faible - qui n'est pas vraiment, selon lui, de l'intertextualité -, faite d'allusions, de simples réminiscences: «chaque fois qu'il y a emprunt d'une unité textuelle abstraite de son contexte et insérée dans un nouveau syntagme textuel, à titre d'élément paradigmatique». M. Riffaterr distingue l'intertextualité aléatoire et l'intertextualité obligatoire. L'intertextualité aléatoire c'est la fonction de la compétence du lecteur: les allusions et les réminiscences d'autres textes qui se trouvent dans le texte. M. Riffaterre préfère consacrer ses études à l'intertextualité obligatoire qui se présente sous forme d'agrammaticalités sémantiques, syntaxiques, morphologiques, et qui nécessite le renvoi à l'intertexte, même si celui-ci n'est pas identifié par le lecteur. Les critiques disent que la distinction entre les deux intertextualités n'existe pas. S'agit-il d'une question de mots: M. Riffaterre n'aurait pas dû parler d'intertextualité aléatoire et obligatoire puisque l'intertextualité est toujours aléatoire, une fonction de la culture du lecteur.

9 Eigeldinger1987:15

10 Greimas 1993: 270 
À partir d'une définition assez large de l'intertextualité comme «croisement de textes», les théoriciens ont voulu se doter d'outils pratiques permettant d'étudier l'intertextualité. Ils se sont heurtés à un grand nombre de questions, notamment celle de la nature du support du renvoi intertextuel, et celle de relations entre microstructure et macrostructure. Les divergences d'opinion sur ces sujets n'ont pas aidé le concept à devenir un réel outil pratique et incontesté.

Il n'est resté de l'intertextualité qu'une définition très large et théorique. Le terme a été porté à l'attention des scientifiques par deux ouvrages publiés en 1989. Le premier était un recueil d'essais intituléIntertextuality in Biblical Writings ${ }^{11}$, contenant à la fois des discussions théoriques et des exemples d'intertextualité biblique. Pour Vorster, l'intertextualité diffère de Redaktionsgeschichte de trois manières importantes : First of all it is clear that the phenomenon text has been redefined. It has become a network of references to other texts (intertexts). Secondly it appears that more attention is to be given to text as a process of production and not to the sources and their influences. And thirdly it is apparent that the role of the reader is not to be neglected in this approach to the phenomenon of text.

Dans le livre «Echoes of Scripture in the Letters of Paul» de Richard Hays ${ }^{12}$ l'auteur présente les relations complexes existant entre les textes de l'Ancien et le Nouveau Testament, car les textes anciens semblent recevoir une nouvelle signification en étant utilisés dans de nouveaux contextes.

Écho intertextuel. Dans son livre Richard Hays parle de l'«écho intertextuel» afin de suggérer que les échos peuvent être assez forts. Les études sur l'Ancien Testament dans le Nouveau représentent les références en forme de citations, allusions et échos. Il n'existe pas de définition exacte mais une citation implique généralement une rupture consciente du style de l'auteur pour introduire des mots provenant d'un autre contexte. Un écho est une faible trace d'un texte et peut être assez inconscient. Il émerge d'esprits imprégnés de l'héritage scripturaire d'Israël. Si un sous-texte est bien connu, la moindre allusion est parfois suffisante pour évoquer sa présence. Un jeu télévisé populaire obligeait les candidats à deviner le titre d'un morceau de musique dans les barres d'ouverture. Parfois, le gagnant le gérait à partir de deux notes seulement. De même, peu de mots sont nécessaires pour évoquer la Pâque ou l'exil en Israël. Les thèmes sont si bien connus et répétés qu'une simple mention apparemment anodine de «doorposts» (dans la langue appropriée) pourrait bien suffisante. Comme le dit Hays à propos des lettres de Paul $^{13}:$ Echoes linger in the air and lure the reader of Paul's letters back

11 Vorster 1989: 15-26

12 Hays 1989: 24

13 Hays 1989: 155 
into the symbolic world of Scripture. Paul's allusions gesture toward precursors whose words are already heavy with tacit implication.

Hays illustre son approche dans les lettres de Philippiens 1:19. La dernière partie du verset est en parfait accord avec le livre de Job 13:16. Hays note que l'écho n'est pas marqué et que les paroles de Paul sont parfaitement compréhensibles sans recours au livre de Job. L'écho invite le lecteur à voir des parallèles entre les deux situations. L'auteur ajoute: «By echoing Job's words, Paul the prisoner tacitly assumes the role of righteous sufferer, as paradigmatically figured by Job... thereby he implicitly transfers to himself some of the significations that traditionally cluster about the figure of Job». L'écho intertextuel n'est pas une typologie. Paul ne suggère pas que sa souffrance est un «accomplissement» du passage de Job. L'écho établit un lien qui invite à la fois des similitudes et des différences. En termes de similitudes, Hays note que la douleur de Job est aggravée par les conseils pieux de ses propres amis, tout comme l'emprisonnement de Paul est aggravé par ceux qui prêchent le Christ (Phlp 1: 15-17). Comme le dit Hays, "the echo whispers a suggestion that the rival preachers have assumed the mantle of Job's hollow comforters; the falsehood of both will be exposed ultimately in the judgment of God».D'autre part, Paul est capable de se réjouir de ses souffrances d'une manière que Job ne pouvait pas, car il est clair que Dieu est à ses côtés. Paul possède ce qui manquait à Job, car sa souffrance est au service du Christ «whereas Job, seeing through a glass darkly, endured his suffering with obdurate puzzlement, Paul, suffering as an apostle of Christ, interprets his suffering as a participation in the sufferings of the crucified Christ and thus finds himself able to rejoice in the midst of adversity»" ${ }^{14}$.

Intertextualité narrative. Dans sa monographie «Paul and his Story. (Re)Interpreting the Exodus Tradition» ${ }^{15}$, Sylvia Keesmaat montre comment l'histoire ou la tradition de l'Exode a influencé l'exégèse de Paul dans les lettres aux Galates et aux Romains. L'histoire de l'Exode dans les écrits de Paul ne se limite pas à ses citations in extenso, mais est activée par toutes sortes d'allusions et d'échos. Keesmaat remarque que Paul ne ressemble pas aux auteurs de Jubilees, car il n'est pas tenu de proposer un récit explicite de l'histoire biblique ou une exégèse systématique du texte donné. Paul s'appuie sur la familiarité de l'histoire de Exode pour formuler ses arguments particuliers. La question n'est pas tant de savoir si Paul invite ses lecteurs à se rappeler un texte en particulier, mais à se souvenir d'une histoire particulière. Le registre intertextuel n'est pas un ensemble de motifs ou de thèmes qui se présentent dans l'esprit collectif du judaïsme du premier siècle. C'est une histoire plus vaste de l'identité et des attentes d'Israël.

14 Hays 1989: 22

15 Keesmaat 1999: 223- 228 
Keesmaat constate que ce qui a été considéré comme des allusions ad hoc dans les lettres de Romains 8: 18-39, est en réalité une relecture minutieuse de l'histoire de l'Exode. Les thèmes clés du passage sont l'adoption, la conduite par l'Esprit, l'appel à Dieu, la souffrance, l'obtention d'un héritage. Celles-ci ont souvent été traitées comme des avantages pour tous les chrétiens, mais lorsqu'il est reconnu que ce sont tous des thèmes clés dans l'histoire de l'Exode, la perspective change. Le péché de la génération du désert était leur désir de retourner en Égypte et de redevenir esclave. Le chrétien inspiré par l'esprit ne doit pas suivre son exemple, mais entrer dans l'héritage promis: «for their own experience of God in Christ Jesus is rooted in the whole story of Israel. Adam and Abraham explain their past, the exodus gives meaning to their present, the whole story provides hope for their future».

Continuité et discontinuité.L'intertextualité narrative implique non seulement une continuité, mais également une discontinuité, car une ancienne histoire est à la fois "perturbée» et «régénérée» lorsqu'elle est utilisée dans des situations nouvelles. Keesmaat met l'accent sur la continuité. Les allusions et les échos de Romains 8: 18-39 ne sont pas choisis au hasard, mais évoquent l'histoire de l'Exode en tant que méta-récit puissant de l'argument de Paul.

Cependant, elle reconnaît également: (a) que l'histoire de l'Exode racontée par Paul en est une qui a déjà été transformée par des prophètes tels qu'Isaiah et Jeremiah; (b) «l'action invasive de Dieu en Jésus-Christ a introduit un nouvel élément dans l'histoire, une tournure inattendue dans l'intrigue»; (c) tout rappel du passé, par opposition à la simple répétition, implique un élément de transformation. L'intertextualité narrative insiste sur le rôle continu d'une histoire significative, tout en reconnaissant que chaque nouvelle narration est en quelque sorte un remodelage de cette histoire.

Intertextualité exégétique. Dans sa monographie «From a broken covenant to circumcision of the heart: Pauline intertextual exegesis in Romans 2:17-29» Timothy Berkley ${ }^{16}$ constate que la clé de la compréhension de l'argument de Paul réside dans l'exégèse des passages particuliers. Berkley reconnaît le travail de Hays sur l'importance de l'écho intertextuel, mais souhaite centrer son étude sur les passages qui trahissent une activité exégétique détaillée. La première partie de l'ouvrage de Berkley est consacrée à l'élaboration des critères permettant d'établir les textes sur lesquels Paul a effectué une exégèse détaillée (Genèse 17, Deutéronome 28 30, Jérémie 7 et 9 et Ézéchiel 36). Il accorde très peu d'importance à la citation explicite d'Isaïe 52: 5 dans les lettres de Romains 2:24, affirmant que ces citations explicites sont des textes de preuve, plutôt que des citations d'activités exégétiques. Pour ce qui est de la stratégie de Paul dans les lettres

16 Berkley 2000: 155 
de Romains 2: 17-29, Berkley affirme que Paul doit montrer que les Juifs ne peuvent compter sur leur statut de peuple de Dieu pour les sauver de la colère. Paul doit traiter les Juifs en tant que peuple bénéficiant des avantages d'un statut spécial devant Dieu. En minimisant la confiance des Juifs dans l'alliance de la loi (2: 17-24), en questionnant la valeur de la circoncision lorsque la loi est violée (2: 25-27) Paul redéfinit la signification de «Juif» pour en faire une définition spirituelle plutôt qu'éthique ou nationale. C'est ainsi que Berkley comprend le passage: la circoncision devient incirconcision parce qu'Israël n'a pas réussi à respecter la loi. Mais Deutéronome, Jérémie et Ézéchiel avaient déjà réinterprété la circoncision de la chair comme signifiant la circoncision du cœur, ou celle qui est intérieure et spirituelle, possible par la foi en Christ. C'est seulement en traçant «l'exégèse intertextuelle» qui se cache dans les lettres de Romains 2: 17-29 que nous pouvons comprendre l'argument de Paul.

Intertextualité dialogique. L'intertextualité narrative insiste sur la continuité, même si elle reconnaît que chaque récit c'est un remodelage de l'histoire. «L'intertextualité dialogique» affirme que le texte source n'est pas toujours aussi malléable comme des catégories traditionnelles telles que l'allégorie ou la typologie. Parfois, le texte source est si puissant qu'il entraîne des associations et des connotations différentes. Davidson ${ }^{17}$ affirme à propos de T S Eliot's, The Waste Land, «The work alluded to reflects upon the present context even as the present context absorbs and changes the allusion». Le terme «l'intertextualité dialogique» suggère que l'influence entre les textes est bilatérale: le nouveau affecte l'ancien, alors que l'ancien affecte le nouveau.

Dans 1'Apocalypse 5, on dit à Jean que «le lion de la tribu de Juda, le descendant du roi David a remporté la victoire» mais lorsqu'il le regarde, il voit «un agneau se tenant comme s'il avait été égorgé» (Ap 5, 4-5). De nombreux spécialistes considèrent cette juxtaposition comme la clé de l'interprétation du livre. Caird dit: «Partout où l'ancien testament dit «Lion», lisez «Agneau»»» ${ }^{18}$. Chaque fois que l'Ancien Testament parle de la victoire du Messie ou du renversement des ennemis de Dieu, nous devons nous rappeler que l'Évangile ne reconnaît pas d'autre moyen d'atteindre ces objectifs que le chemin de la Croix. John Sweet affirme que «le lion de Juda, l'attente messianique traditionnelle, est réinterprété par l'Agneau: la puissance et la victoire de Dieu résident dans le sacrifice de soi» ${ }^{19}$. Il semblerait que l'agneau ait repris des traits guerriers du lion. Par exemple, dans le chapitre suivant, on dit que les peuples du monde se cachent de la «colère de l'agneau»(Ap 6, 16). Ses ennemis reçoivent un double pour leurs péchés et «seront tourmentés de feu et de soufre en présence des saints

17 Davidson 1985: 117

18 Caird 1984: 318

19 Sweet 1990: 125 
anges et de l'Agneau» (Ap 14.10). Il y a une bataille dans Apocalypse 17, mais le résultat n'est pas douteux, car «l'Agneau les vaincra, car il est le Seigneur des seigneurs et le Roi des rois» (Ap 17, 14). Comme le constate Resseguie $^{20}$, «l'Agneau, bien que n'étant pas de nature un animal fort, est un être d'une force indiscutable dans ce livre». «L'intertextualité dialogique» vise à décrire un état de choses dynamique plutôt que statique. L'utilisation de la juxtaposition lion / agneau par John n'est pas résolue en déclarant que l'agneau réinterprète le lion, car il faut également rendre compte de la façon dont le lion a affecté l'agneau.

Intertextualité postmoderne. Dans l'ouvrage «Intertextuality in Biblical Writings, Ellen van Wolde» ${ }^{21}$ décrit la manière dont un texte est produit: The writer assigns meaning to [their] own context and in interaction with other texts... shapes and forms [a] text. The reader, in much the same way, assigns meaning to the generated text in interaction with other texts [they] know... A writer does not weave a web of meanings that the reader merely has to follow, but... presents them to the reader as a text. The reader reacts to the offer and enters into a dialogue with the possibilities the text has to offer.

La lecture comporte un élément subjectif car «toutes les interprétations doivent nécessairement délimiter les références possibles d'un texte pour donner un sens cohérent»" ${ }^{22}$. Chaque «texte - en tant qu'intersection d'autres surfaces textuelles - suggère un surplus indéterminé de possibilités significatives».

Vernon Robbins note que la plupart des exemples d'intertextualité biblique ont pris des décisions fondamentales, telles que: donner la priorité aux textes juifs plutôt qu'aux textes grecs ou romains; souligner l'influence des textes sur d'autres expressions de la culture; se limiter aux modes de discours historiques et littéraires. Ainsi, «la nature idéologique de toute interprétation se manifeste dans l'interaction entre le choix d'un mode du discours interprétatif et le choix des dimensions du texte que l'interprète réinscrit» ${ }^{23}$

Selon Schneiders, la lecture «traditionnelle» voit Jésus (le mâle) en conversation avec une femme de Samarie mais opérant à un niveau différent (plus élevé). Son esprit est fixé sur les questions terrestres. Elle est venue au puits chercher de l'eau ordinaire (4: 7). Quand Jésus explique: «Tous ceux qui boivent de cette eau auront de nouveau soif, mais ceux qui boivent de l'eau que je leur donnerai n'auront plus jamais soif», son intérêt est éveillé mais uniquement pour se sauver du voyage quotidien (4: 15). Lorsque Jésus expose le fait qu'elle a eu cinq maris, elle essaie de le

20 Resseguie 1998: 129

21 Wolde 1989: 43-49

22 Beal 1992: 27-39

23 Robbins 1996: 296 
provoquer dans une discussion théologique sur les lieux de culte. L'adoration ne concerne pas le lieu, mais l'esprit et la vérité (4:24) «Dieu est Esprit et ceux qui l'adorent doivent l'adorer en étant guidés par son Esprit et selon la vérité». Enfin, elle énonce un principe de base de ses croyances samaritaines: "Je sais que le Messie arrive ... Lorsqu'il viendra, il nous expliquera tout» (4.25). Jésus répond: «Je suis celui qui te parle» (4.26). Schneiders représente la femme comme un mécréant peu recommandable (bien qu'intéressant) qui s'abandonne à son immense connaissance surnaturelle d'elle ${ }^{24}$.

Cependant, une lecture différente est possible, car c'est Jésus qui demande un verre littéral. La femme ne pense qu'en termes terrestres; Jésus est assis près du puits de Jacob et lui demande un verre matériel. Mais la femme regarde au-delà de la matière pour se demander pourquoi les tabous sont ignorés car «les Juifs ne partagent pas de points communs avec les Samaritains» (4: 9). Jésus répond qu'il peut offrir «de l'eau vive», que la femme prend (à juste titre) comme prétention religieuse d'être supérieure à Jacob et aux patriarches. Jésus précise ensuite que l'eau qu'il offre est telle que ceux qui en boivent n'en auront plus jamais soif, car elle «deviendra en eux une source d'eau jaillissant pour la vie éternelle». La femme est intéressée et répond en utilisant la même métaphore que celle de Jésus: "Monsieur, donnez-moi cette eau, afin que je n'aie plus soif ou que je n'aie plus besoin de venir ici pour puiser de l'eau" (4:15). Il est donc possible de lire le texte comme un échange théologique sérieux et non comme une femme qui ne comprend toujours pas le mâle (supérieur). Cependant, étant donné la nature symbolique d'une grande partie de l'Évangile selon Jean, il faut bien admettre que c'est «une possibilité». Si le lecteur était supposé reconnaître que «Détruisez ce temple et que dans trois jours je le rebâtirai» (Jn 2, 19) faisait référence au «temple de son corps». Il serait certainement possible qu'on se demande si Jérusalem ou Gérizim est le lieu approprié pour le culte (le lecteur devrait connaître l'histoire des Samaritains).

Stephen Moore note qu'il y a des interprétations contradictoires. Il existe des gens qui souhaitent condamner la femme et ceux qui ne peuvent le faire ${ }^{25}$. Ils ne peuvent la condamner que s'ils participent à son erreur, ne peuvent lui attribuer une histoire d'immoralité qu'en lisant «charnellement» qu'elle le fait - la lecture littérale de 4:18 risque de devenir une reconstitution déplacée d'un autre Épisode de Johannine, dans lequel une femme sans nom est accusée d'immoralité sexuelle par des accusateurs qui sont eux-mêmes accusés (8:11).

«L'intertextualité postmoderne» attire l'attention sur le fait qu'il existe toujours divers façon d'interpréter un texte. L'intertextualité se révèle

24 Schneiders 1991: 194

25 Moore 1994: 142 
comme un concept fructueux et créatif. Elle invite le lecteur à se concentrer sur la complexité textuelle. Des études intertextuelles suggèrent que les allusions et les échos pourraient être aussi importants que des citations explicites pour la compréhension d'un texte. Certains pensent que cela introduit un domaine d'étude plus spéculatif que les citations explicites, car les allusions et les échos sont naturellement plus insaisissables que les citations.

Les spécialistes utilisent le terme «intertextualité» de manières très différentes et, dans certains cas, incompatibles. Certains l'utilisent notamment pour rassembler des preuves à l'appui d'une interprétation particulière (Berkley), tandis que d'autres l'utilisent pour montrer les tensions inévitables et la complexité inhérentes à l'utilisation de l'Ancien Testament dans le Nouveau Testament ${ }^{26}$.

Aichele et Phillips aimeraient limiter l'utilisation de ce terme à ses racines postmodernes et considérer les utilisations plus «conservatrices» ${ }^{27}$. D'autre part, le mot «intertextualité» a pris une vie propre et devrait être interprété à la lumière de la pratique actuelle plutôt que du moment originel.

La question de la relation entre intertextualité et traduction a été réduite par Genette à une relation d'inclusion: parmi les divers types de relations intertextuelles au sens large figure la relation hypertextuelle qui unit deux textes:l'hypertexteest dérivéde l'hypotexte. La traduction d'après cette définition, est une forme d'hypertextualité, mais il en est bien d'autres.

Chez Berman, l'hypertextualité est réinterprétée comme l'un des modes de la traduction, mode qu'il rejette au même titre que la traduction «ethnocentriste».Si l'intertextualité intervient pour conditionner différents aspects du sens et de l'effet communiqués par le texte, on peut facilement imaginer que, dans le cas d'une lecture différée et, plus encore, dans celui d'une traduction, la réception du texte par un lecteur réel éloigné des caractéristiques du lecteur implicite va changer les conditions du jeu intertextuel. Est-il possible de traduire un texte sachant qu'une partie du sens et de l'effet se construit en fonction d'autres textes? Si «l'intertextualité parle une langue dont le vocabulaire est la somme des textes existants», peut-on traduire un texte écrit dans cette langue, dont le dictionnaire est toute une bibliothèque? La question suggère de rechercher les différents facteurs qui rendent l'opération facile ou difficile, voire possible ou impossible.

Pour décrire, dans le texte original, les lieux d'intertextualité, il convient de se munir de quelques définitions, que nous emprunterons pour l'essentiel à Genette. On appellera «trace intertextuelle» une séquence du texte qui peut être reconnue comme provenant d'un autre texte. Cette

26 Moyise 2000: 14-41

27 Aichele \& Phillips1995: 11 
réapparition peut être littérale, le retour du déjà dit ne comportant aucune transformation du signifiant d'origine; au contraire le signifiant d'origine peut être transformé, tout en restant identifiable. Par ailleurs, le réemploi peut être déclaré: la séquence empruntée est alors accompagnée d'une ou plusieurs marques explicites de son origine allographe (nom d'auteur, titre d'œuvre, guillemets, etc.). Il peut ne pas l'être: Genette utilise dans ce cas le terme de «plagiat». En croisant ces deux axes, on voit se dessiner quatre types de traces intertextuelles.La citation est un emprunt littéral et déclaré, la forme la plus visible de l'intertextualité, notamment grâce à des codes typographiques (décalage de la citation, restitution de la présentation typographique du texte cité, emploi des caractères italiques ou des guillemets). La référence est un emprunt non littéral mais déclaré. L'allusion est un emprunt non littéral et non déclaré. L'allusion complète la citation: "Une citation proprement dite n'est jamais que la preuvre d'une érudition facile et commune ; mais une belle allusion est quelquefois le sceau du génie". L'allusion repose sur l'implicite et suppose que le lecteur comprenne qu' il s'agit d' un jeu de mots ou d'un clin d'œil.

Atteinte à la propriété littéraire, il constitue un emprunt à un texte littéraire sans que ses références soient explicitement indiquées - le plagiat. Pour éviter «le plagiat», on parle pour le quatrième type d'emprunt - littéral et non déclaré. Face à la traduction, ces quatre types de traces ont des comportements relativement différents. La traduction d'une référence se ramène souvent à la transcription de noms propres, en respectant l'usage de la langue d'arrivée. La traduction d'une citation doit respecter non seulement le sens du fragment cité, mais l'effet qu'il produit. La citation traduite n'a souvent aucun contexte sinon simulé, elle est pur trompe-l'œil et ne peut donc se permettre d'être stylistiquement déficiente. Certains traducteurs résolvent parfois le problème de la traduction de la citation en passant en mode non littéral, c'est-à-dire en remplaçant la citation par une paraphrase, assortie ou non d'une référence. L'allusion présente pour le traducteur plusieurs types de difficultés qui peuvent se superposer. Même en présence d'une référence, le texte qui contient la solution de l'énigme n'est pas littéralement présent dans le texte citant et ne peut donc pas être traduit. Il y a donc dispersion du sens sur deux lieux, dont l'un prend en charge la trace explicite, tandis que l'autre explicite le non-dit. La traduction d'un emprunt non déclaré semble comporter moins de contraintes.

Les difficultés de traduction et les méthodes employées pour les résoudre sont donc variables selon la forme de la trace. Un autre type de problème concerne non plus la trace elle-même, mais son mode d'intégration dans le contexte du texte citant. La trace intertextuelle, quelle que soit sa nature, s'intègre parfois, dans la phrase ou le passage qui la contient, sans créer de perturbation majeure au niveau du sémantisme. Une traduction relativement proche du texte original, à défaut de restituer le sens 
intégral, est du moins compréhensible. Mais la trace peut aussi s'insérer de façon telle que seul le recours au texte cité permette de construire un sens satisfaisant pour l'ensemble du passage citant. Sans ce recours, le passage est inintelligible: selon Genette, il est «non recevable» et Riffaterre parle d'une anomalie sémantique qu'il appelle «agrammaticalité»; pour l'un et l'autre, cet accident sémantique est le signe le plus fréquent de la présence d'une allusion. Le sens ne peut être rendu que par une solution qui prendrait en compte à la fois le texte citant et le texte cité et qui restituerait conjointement le sens et l'effet: seule une libre recréation du traducteur pourrait relever le défi.

La traductiond'un passage intertextuel ne dépend pas seulement du texte citant, mais aussi du texte cité. Dans la problématique de l'intertextualité, l'expression «texte cité» doit être comprise au sens large: texte écrit, mais parfois texte non écrit (discours social, sagesse populaire, discours idéologique) ou système signifiant non verbal (iconographie, musique).

L. Jenny distingue une intertextualité «faible», qui peut être simplement la revendication d'un ancrage dans un auteur, une culture ou une idéologie, et un véritable conflit, le texte cité étant alors considéré comme représentant d'une pensée, d'une poétique ou d'une idéologie surannée, pesante ou dangereuse: le conflit dont le texte cité ne sort pas indemne et dont l'issue constitue le véritable enjeu, la visée du texte citant. Pour M. Riffaterre, plus radical, il n'y a intertextualité que lorsque ce conflit entre «codes antagonistes» peut être repéré.

L'intertextualité permet une gestion très économique du sens: le texte produit un maximum de sens avec un minimum de signifiant. Mais, pour ce faire, elle suppose la coopération du lecteur: le sens du texte citant ne lui sera donné, l'effet ne lui sera sensible que dans la mesure où il sera lui-même en état d'apporter la connaissance du texte cité. Il faut donc faire intervenir la notion de «compétence intertextuelle» du lecteur réel, celle-ci étant définie comme la connaissance, antérieure à la lecture du texte citant, des textes cités et des moyens habituels d'y faire référence. Si nous transposons au cas des «sujets lisants» l'analyse des compétences des «sujets parlants» proposée par C. Kerbrat-Orecchioni, nous dirons que ces deux aspects ressortissent aux deux catégories appelées par cet auteur «compétence encyclopédique» et «compétence rhétorico-pragmatique» ${ }^{28}$

Lorsque le lecteur réel n'est pas très différent du lecteur implicite, cette coopération est désirable. Mais qu'en est-il dans le cas du texte traduit, reçu par un lecteur ayant un tout autre univers de référence et disposant d'une tout autre compétence? Quand on traduit un texte, on ne traduit pas en même temps sa «bibliothèque»; une fois traduit, le texte peut

28 Kerbrat-Orecchioni 1986: 27 
donc se retrouver très loin d'elle: décontextualisé. Passage par passage, le travail du traducteur sera conditionné par le degré de la décontextualisation, c'est-à-dire par la distance faible, moyenne ou immense, entre culture d'accueil et texte cité. La décontextualisation est faible lorsque la présence culturelle du texte cité est identique ou analogue dans les deux cultures mises en contact par la traduction; c'est le cas de cultures proches ayant des zones de références communes. Un emprunt à la Bible pose généralement peu de problèmes lors d'une traduction entre langues européennes, chacune des cultures correspondantes ayant, malgré des nuances, un même ancrage dans le texte sacré; une version de la Bible dans la langue nationale peut être considérée comme familière au lecteur. Ainsi, la traduction d'un emprunt biblique se ramène souvent à une simple recherche de correspondance.

Si l'intertextualité intervient pour conditionner différents aspects du sens et de l'effet communiqués par le texte, on peut facilement imaginer que, dans le cas d'une lecture différée et, plus encore, dans celui d'une traduction, la réception du texte par un lecteur réel éloigné des caractéristiques du lecteur implicite va changer notablement les conditions du jeu intertextuel. Est-il possible de traduire un texte sachant qu'une partie du sens et de l'effet se construit en fonction d'autres textes? Peut-on traduire l'intertextualité? L'analyse a mis en évidence l'existence d'une «marge» de sens ou d'effet qui, dans les conditions normales semble échapper à la traduction. En effet, du fait de la décontextualisation, la conservation intégrale du sens découlant de l'insertion, sur le mode de la synecdoque, de tout un texte allographe ne paraît guère possible: le texte traduit n'utilise pas les ressources de la même langue, il n'évolue pas dans le même univers de référence, il n'est pas destiné au même lecteur. Une fois traduit, le texte peut signaler l'intertextualité de son texte directeur, il peut aussi l'expliciter, ou encore la simuler avec d'autres moyens, mais il ne peut pas à la fois conserver le même fonctionnement et produire le même effet. Il semble donc qu'il faille compter avec une certaine entropie, l'habileté ou la créativité du traducteur, mais qui, d'un point de vue théorique, ne peut être nulle.

L'intertextualité et la traduction. Le texte traduit ne peut pas proposer une réplique à l'identique de l'intertextualité de son texte directeur, mais qu'il occupe une position forcément autonome. C'est le positionnement intertextuel d'une traduction qui détermine si cette traduction est vivante, lisible, ou si elle est désuète, inerte. L'activité traductive occupe une place capitale au nombre des pratiques qui assurent la constitution du champ de l'intertextualité. Le texte traduit, bien plus qu'un texte non traduit, contribue à enrichir le champ intertextuel de sa culture d'accueil. Ce sont les traductions qui créent des ponts entre les cultures du monde, qui densifient les réseaux déjà existants. C'est par leur jeu que se constitue cet ensemble constamment en train d'être réévalué, ce domaine en 
perpétuel mouvement, animé de glissements et de rebonds, de soubresauts et d'échos, cette immense toile frémissante qui est la bibliothèque de l'humanité.

La notion d'intertextualité est utilisé pour décrire les liens qui existent entre des textes. Les linguistes parlent d'intertextualité verticale, un phénomène par lequel un texte d'un médium, ou moyen de communication, influence un texte d'un autre médium. Dans l'intertextualité horizontale, un texte dans le même médium peut en influencer un autre. Paul Ricœur qualifie la Bible comme «le plus grand intertexte vivant» ${ }^{29}$ qui est à l'origine de nombreuses œuvres et de diverses théories de l'interprétation ${ }^{30}$. Dans la Bible il s'agit d'intertextualité horizontale où un texte peut servir d'arrière-plan à un autre. En façonnant un nouveau texte, un écrivain peut faire référence à d'autres textes, d'une manière consciente ou inconsciente, que ce soit reconnu par le lecteur ou non.

Pour examiner l'intertextualité biblique, nous adopterons une acception plus restreinte du terme en tant que référence conventionnelle à une relation de coprésence entre différents textes concrétisée dans une réécriture ou par le biais de la citation, de la référence ou de l'allusion visant à envisager un jeu d'échos, de continuités ou d'écarts. Si l'on considère quetout texte est considéré comme un palimpseste qui permet d'entrer en communication avec lamémoire littéraire, toute textualité serait à entendre comme intertextualité. Le critique canadien Northtop Frye résume sa conception del'intertextualité par le sophisme post hoc ergo propter hoc en réponse aux critiques littéraires n'admettant pas la Bible comme source culturelle et civilisationnelle essentielle. L'on ne pourrait, en d'autres termes, omettre de traiter l'influence de la Bible sur l'imagination et son impact sur les créations artistiques ${ }^{31}$.

Pour le lecteur biblique, l'interdépendance entre les deux testaments est évidente. L'Ancien Testament est souvent cité mot pour mot, ses références se trouvant sur presque chaque page du Nouveau Testament. Mais certains ignorent que ces textes de l'Ancien Testament ne proviennent pas du texte en hébreu, mais de la traduction grecque. Par exemple, dans le premier évangile, Matthieu cite des promesses de l'Ancien Testament pour démontrer d'une manière systématique que Jésus-Christ est le Messie, l'envoyé promis par Dieu à son peuple. Les propos de Jésus cités par les quatre évangélistes montrent que la pensée et la parole du Messie sont imprégnées de ces textes anciens, à tel point que certains sont tentés de demande qu'est-ce que Jésus a dit de nouveau? Dans les paroles de JésusChrist rapportées dans le Nouveau Testament, on trouve les traces des écrits de l'Ancien Testament. En lisant les Évangiles, on a l'impression que Jésus

29 Marguerat et Curtis 2000: 9

30 Panier 1997: 314

31 Northrop 1984: 32 
cite ces écrits par cœur. Mais ce n'est pas seulement dans le Nouveau Testament que l'on constate cette interdépendance des textes. Dans l'Ancien Testament les auteurs avaient accès aux livres écrits par les autres auteurs ou à une tradition orale partagée par la communauté (l'histoire de la création, la vie des premiers hommes, les évènements majeurs dans la vie des patriarches etc.)

Concernant l'interdépendance des œuvres bibliques, il est important de faire une distinction entre deux sortes de références: les citations directes (les mots du prophète Ésaïe, cités dans les Évangiles et les Épîtres) et les allusions (des références indirectes aux mots ou aux images d'un texte à l'intérieur d'un autre).

Dans la Bible, beaucoupde citations directes de textes bibliques se trouvent dans le Nouveau Testament. Les citations directes de l'Ancien Testament dans le Nouveau sont introduites par des formules quiindiquent la source de citation ou l'auteur (lorsqu'il s'agit d'un prophète) Voici, j'envoie mon messager en avant de toi, pour préparer ton chemin. Une voix crie dans le désert: Préparez le chemin du Seigneur, rendez droits ses sentiers. (Marc 1.2-3 citant És 40.3); Celui qui n'était pas mon peuple, je l'appellerai Mon Peuple et celle qui n'était pas la bien-aimée, je l'appellerai Bien-Aimée..(Rom 9.25 citant Osée 2.25)

Une allusion est la manière d'éveiller l'idée d'une personne ou d'une chose sans en faire expressément mention. Ce procédé stylistique est très courant où les allusions peuvent provenir de tous les genres de textes bibliques. Martin Luther King utilise des allusions bibliques dans ses discours. Il dit: "J'ai été sur la montagne», allusion à Moïse qui perçoit la Terre Promise de loin d'en haut d'une montagne, sans avoir jamais pu y entrer (Deut 32.48-50). Ces mots sont presque prophétiques, car comme Moïse, il aide les Noirs Américains, à trouver la liberté, sans pour autant en jouir lui- même.

Le message de la voix venant du ciel en Marc 1.11 est une allusion: «Tu es mon Fils bien-aimé, il m'a plu de te choisir»cette phrase évoque le souvenir d'un passage de l'Ancien Testament, le Psaume 2.7: le Seigneur m'a dit: "Tu es mon fils; moi, aujourd'hui, je t'ai engendré». Cette intertextualité est omniprésente dans le Nouveau ou l'Ancien Testament.

Dans le Psaume 23: Le Seigneur est mon berger, les allusions bibliques sont basées sur d'autres lignes du psaume: Je ne manquerai de rien... Il me fait reposer dans de verts pâturages. Les citations et les allusions intertextuelles enrichissent un texte, renforcent la perception. Mais ils posent les problèmes pour la traduction: au niveau des termes clés, des liens entre les citations et leur contexte, au niveau du contenu et du style des textes eux-mêmes.Le traducteur doit traiter avec attention les références à l'Ancien Testament au sein du Nouveau. Il faudrait garder intacts les termes clés. Dans le livre des Actes, un passage de Joël est cité (2.20): Le soleil se 
changera en ténèbres et la lune en sang avant que vienne le jour du Seigneur, grand et glorieux. Le traducteur rend l'expression «jour du Seigneur» d'une manière moins littérale «avant que le Seigneur n'arrive» ou «avant qu'il ne vienne». Mais l'expression le jour du Seigneur est une notion qui apparaît dans la Bible dans d'autres contextes: Vous-mêmes le savez parfaitement: le jour du Seigneur vient comme un voleur dans la nuit. Il faudrait garder une traduction plus conservatrice des citations directes pour que le lecteur puisse voir le lien entre les références, d'autres passages et avec son texte d'origine: Quel jour! Il est proche, le jour du Seigneur il vient du Dévastateur, comme une dévastation (Joël 1.15)

Le traducteur doit s'assurer que le lien qui existe entre le texte cité et le passage où celui-ci se trouve soit clair. Par exemple, lorsque Paul cite le prophète Ésaïe 29.14 dans sa première lettre à l'Église de Corinthe (1 Cor 1.19-21): Je détruirai la sagesse des sages et j'anéantirai l'intelligence des intelligents. Où est le sage? Dieu n'a-t-il pas rendue folle la sagesse du monde? En effet, puisque le monde, par le moyen de la sagesse, pas connu Dieu dans la sagesse de Dieu, c'est par la folie de la prédication que Dieu a jugé bon de sauver ceux qui croient.(1 Cor 1.19-21). Les mots «sage» et «sagesse» apparaîssent dans le passage qui suit. (1.22, 24, 25, etc.). Si le traducteur ne se rend pas compte de la démarche de Paul: citation + commentaire, il peut fausser le sens de ce texte. Il est important de garder la cohérence du texte et de la pensée de Paul, en gardant le lien entre le mot «sage» et «sagesse» dans la citation et dans le texte qui suit. Dans les Livres Saints, on lit: «Je détruirai la sagesse des sages et je rejetterai l'intelligence des gens intelligents» Qu'est-ce que le sage peut dire encore/ Dieu a montré que la sagesse de ce monde est une folie. En effet, le monde avec sa sagesse n'a pas reconnu Dieu en voyant la sagesse de Dieu. Les traducteurs aurait pu rendre les mêmes idées d'une manière plus dynamique, mais ils conservent le lien entre la citation et son contexte.

Les traducteurs remarqueront que le texte cité dans le Nouveau Testament n'est pas le même que celui de l'Ancien Testament. Dans l'exemple d'Ésaïe 29.14, les passages ne sont pas identiques, même dans une version plus formelle. Le texte de l'Ancien Testament dit: C'est pourquoi je vais continuer à lui prodiguer des prodiges, si bien que la sagesse des sages s'y perdra, et que l'intelligence des intelligents se dérobera. On peut constater les différences entre le texte d'origine et celui cité dans l'épître aux Corinthiens. Paul cite la forme grecque du verset, et non pas la forme de l'hébreu. Et ceci est le cas pour les citations de l'Ancien Testament dans le Nouveau. Au moment où le Nouveau Testament a été rédigé, la version grecque de l'Ancien Testament, était plus connue que la version en hébreu. La version grecque est une traduction qui est susceptible de comporter des devers adaptations. 
Le traducteur, devrait il traduire le texte du Nouveau Testament tel qu'il est en grec ou harmoniser les textes du Nouveau Testament avec ceux de l'Ancien? Ésaïe a écrit sa prophétie concernant «la jeune femme qui sera enceinte», le mot désignant une jeune personne voulait dire «jeune fille». Matthieu interprète ce mot comme «vierge» (en grec).

Dans la traduction du Nouveau Testament, les problèmes de l'intertextualité sont nombreux. La traduction des passages avec des citations ou des allusions est plus difficile que la traduction des textes simples. Le traducteur doit considérer la Bible comme l'origine de diverses théories de l'interprétation, d'un ensemble de textes.

$\mathrm{Au}$ terme de ces réflexions sur le rapport entre intertextualité et traduction, il apparait, d'une part, que le texte traduit ne peut pas proposer une réplique à l'identique de l'intertextualité de son texte directeur, mais qu'il occupe, dans un champ intertextuel forcément autre, une position forcément autonome.

L'activité traductive occupe une place capitale au nombre des pratiques qui assurent la constitution du champ de l'intertextualité. Le texte traduit, bien plus qu'un texte non traduit, contribue à enrichir le champ intertextuel de sa culture d'accueil. Ce sont les traductions qui créent des ponts entre les cultures du monde, qui densifient les réseaux déjà existants. C'est par leur jeu que se constitue cet ensemble constamment en train d'être réévalué, ce domaine en perpétuel mouvement, animé de glissements et de rebonds, de soubresauts et d'échos, cette immense toile frémissante qui est la bibliothèque de l'humanité.

\section{Bibliography:}

Aichele \& Phillips 1995 = George Aichele and Gary A. Phillips, Intertextuality and the Bible. Introduction: Exegesis, Eisegesis, Intergesis, Semeia 69-70. Atlanta, GA: Scholars, 1995.- pp.7-18.

Angenot 1983 = Marc Angenot, L'intertextualité : enquête sur l'émergence et la diffusion d'un champ notionnel». Revue des sciences humaines tome LX ${ }^{\circ}$ 189, janvier-mars 1983, pp. 121-135.

Barthes 1973 = Roland Barthes, Le Plaisir du texte. Paris.: Edition du Seuil.,1973. $110 \mathrm{p}$.

Beal 1992 = Timothy K. Beal, Ideology and intertextuality: Surplus of meaning and controlling the means of production, in: D N Fewell, (ed), Reading between texts: Intertextuality and the Hebrew Bible, Louisville: Westminster John Knox. pp. 27-39.

Berkley $2000=$ Timothy Wayne Berkley, From a broken covenant to circumcision of the heart: Pauline Intertextual Exegesis in Romans 2:17-29. Atlanta: SBL. - Marquette University. (SBL Dissertation Series 175)., 2000.- 160 p. 
Caird $1984=$ George Bradford Caird, The Revelation of St John the divine. London: A \& C Black, (2nd Ed), 1984.- 318 p.

Davidson 1985 = Harriet Davidson TS, Eliot and hermeneutics: Absence and interpretation in the Waste Land. / - Louisiana State University Press. Baton Rouge., 1985. - 117 p.

Eigeldinger 1987 = Marc Eigeldinger, Mythologie et intertextualité. Genève.: Slatkine, 1987. - $15 \mathrm{p}$.

Foucault, Barthes, Derrida $1968=$ Michel Foucault, Roland Barthes, Jacques Derrida, M. Théorie d'ensemble. Collection "Tel Quel". Paris.: Edition du Seuil,1968. - 410 p.

Genette 1982 = Gérard Genette, Palimpsestes. La littérature au second degré. Paris.: Edition du Seuil, 1982.- pp. 7-14.

Greimas, Courtès 1993 = Algirdas Julien Greimas, Joseph Courtes,Sémiotique. Dictionnaire raisonné de la théorie du langage. Paris. : Hachette, 1993. - 270 p.

Hays1989 = Richard B. Hays, Echoes of scripture in the letters of Paul. New Haven: Yale University Press, 1989. - 240 p.

Keesmaat 1999 = Sylvia C.Keesmaat, Paul and his Story: $(\mathrm{Re})$ Interpreting the Eodus Tradition. (JSNTSup 181). Sheffield: Sheffield Academic Press, 1999. - 289 p.

Kerbrat-Orecchioni $1986=$ Catherine Kerbrat-Orecchioni, L'implicite. Paris.: A. Colin, 1986.- pp.44-47.

Kristeva 1969 = Julia Kristeva, Recherches pour une sémanalyse. Paris.: Ed. duSeuil, 1969. - 146 p.

Kristeva 1969 = Julia Kristeva, «Le mot, le dialogue et le roman», Sèméiotikè. Recherches pour une sémanalyse. Paris.:Ed. duSeuil, 1969. - pp.82-112.

Maingueneau 1976 = Dominique Maingueneau, Initiation aux Méthodes de l'analyse du discours. Paris.: Hachette, 1976. - 191 p.

Marguerat et Curtis $2000=$ Daniel Marguerat et Andrian Curtis, Intertextualités. La Bible en échos. (coll. Le Monde de la Bible n 40). Genève.: Labor et Fides., 2000. - $200 \mathrm{p}$.

Moore 1994 = Stephen D. Moore, Poststructuralism and the New Testament: Derrida and Foucault at the foot of the cross. Minneapolis: Fortress Press, 1994.-143 p.

Moyise $2000=$ Steve Moyise, Intertextuality and the study of the Old Testament in the New Testament, in: Steve Moyise, (ed), The Old Testament in the New Testament (JSNTSup189). Sheffield: Academic Press.,2000. - pp.14-41.

Northrop 1984 = Northrop Frye, Le Grand code: la Bible et la littérature. Traduit de l'anglais par Catherine Malamoud. Edition du Seuil, coll. Poétique.,1984, $-32 \mathrm{p}$.

Panier 1997 = Louis Panier, «Du texte biblique à l'énonciation littéraire et à son sujet», La Bible en littérature. Actes du colloque international de Metz, 
publiés sous la direction de Pierre-Marie Beaude. Université de Metz.: Cerf.,1997. - pp. 313-326.

Resseguie 1998 = James L. Resseguie, Revelation Unsealed: A Narrative Critical Approach to John's Apocalypse. (Biblical Interpretation). Leiden: Brill., 1998. - 233 p.

Riffaterre 1979 = Michel Riffaterre, Sémiotique intertextuelle:l'interprétant. Revue d'esthétique $\mathrm{n}^{\circ} 1-2 ., 1979$. - pp. 128-150.

Robbins 1996 = Vernon K.Robbins, The tapestry of early Christian discourse: Rhetoric, society and ideology. London: Routledge., 1996. - 296 p.

Schneiders $1999=$ Sandra M. Schneiders, The revelatory text: Interpreting the New Testament as sacred scripture. San Fransisco: Harper San Fransisc., 1999. $248 \mathrm{p}$.

Sweet $1990=$ John Sweet, Revelation. TPI New Testament Commentaries, 2 nd ed. London: SCM., Press Philadelphia:Trinity Press International., 1990. - 125p.

Van Wolde 1989 = Ellen Van Wolde, Trendy Intertextuality, in Sipke Draisma (ed), Intertextuality in Biblical Writings. Kampen: Kok, 1989. - pp. 43-49.

Vorster $1989=$ Willem Vorster, Intertextuality and Redaktionsgeschichte, in Sipke Draisma (ed), Intertextuality in Biblical Writings. Kampen: Kok, 1989. - pp. 15-26. 